\title{
PARAMETERIZEd Finite Element ANALYSis With OPTIMIZATION OF A SUPERPlastic ForMING Process USING ANSYS®
}

\author{
SALEM, N.
}

Abstract: This paper is an approach, from the optimization techniques, the point of view, provided by Ansys ${ }^{\circledR}$ for a superplastic deformation process of a commercial Al and $\mathrm{Cu}$ alloy called SUPRAL 100 (Aluminum 2004). In the present study, a finite element analysis, followed by application of some software optimization features were carried out to predict the deformation mode in various predetermined conditions and to optimize the forming process and enhance the product quality. In this regard, were established two input parameters(Sample_diameter and Displacement $Y$ Component) and two output parameters(Equivalent Stress Maximum and Total Deformation Maximum) to study how the latter are influenced by the former and which the optimization design effort magnitude is. After the finite element analysis was carried out, all optimization methods available in Ansys ${ }^{\circledR}$, Design Exploration module, were applied(Direct Optimization, Response Surface Optimization, and Six Sigma Analysis) and results were studied as a measure of parameters influence, using Ansys ${ }^{\circledR}$, Parameters Correlation, module. This study should be regarded as consisting of two separate analyses, but that complement each other: a finite element analysis of superplastic deformation and an analysis of the opportunities to optimize the forming process, respectively.

Key words: Optimization Finite Element Analysis, Superplasticity and Superplastic Forming, Engineering Design, Simulation Of The Plastic Deformation Processes
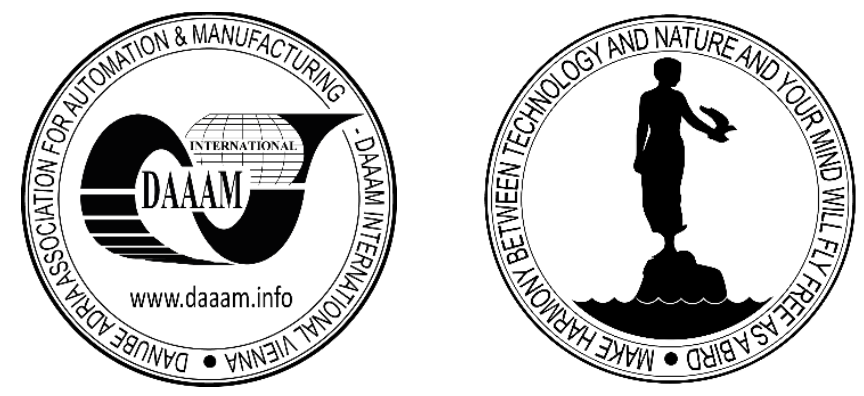

Authors' data: Salem, N[azzal], Zarqa University, Po Box 132222, Zarqa, Jordan, nsalem@zu.edu.jo

This Publication has to be referred as: Salem, N[azzal] (2017). Parameterized Finite Element Analysis With Optimization of a Superplastic Forming Process Using ANSYS ${ }^{\circledR}$, Chapter 24 in DAAAM International Scientific Book 2017, pp.319-332, B. Katalinic (Ed.), Published by DAAAM International, ISBN 978-3-902734-12-9, ISSN 1726-9687, Vienna, Austria

DOI: $10.2507 /$ daaam.scibook.2017.24 
Salem, N.: Parameterized Finite Element Analysis With Optimization of a Superpla...

\section{Introduction}

The engineering system design is an interdisciplinary process that requires cooperation between designers in different engineering fields. That is why engineering design is considered to be a complex process, and it must be approached very carefully. Engineering design requires hypotheses that need to be adopted for the development of models that can be subjected to analysis, verification, and experiments. The design begins with the analysis of several variants. For most applications, the entire design project can be subdivided into different subproblems that can then be independently addressed. Each subproblem can thus be presented as a design optimum to be mathematically solved, Diehl, M., Glineur, F., Jarlebring, E. \& W. Michiels, [1].

Numerical methods for solving optimization problems are also known as the mathematical programming framework. This framework provides a generally flexible formulation to solve engineering design issues, Belegundu, A. D. \& Chandrupatla, T. R. [2].

Sometimes optimization issues in engineering design may not have solutions, or they are totally inappropriate, with improper values. This is due, in general, to the existence of a mathematical conflict, since the problem may be incorrectly formulated. For example, it is possible that the formulated restrictions make it impossible to find a limited area of the test space, or that this space is undefined due to unlimited or mathematically indefinite restrictions. These accidents should be treated with care, without generalist approaches.

Frequently, the simulation of the plastic deformation processes through the finite element method has proved useful and efficient. This method is extremely useful in exploration the results of several candidate processes. Wherefore, the method becomes, in fact, an original optimization algorithm, since the calculations are based on input parameters such as geometry, mesh analysis, strengths, constraints, material model, friction, non-linear behavior laws of the material. This approach, to the finite element method in the analysis of plastic deformation processes, has reached a certain degree of maturity. After the finite element analysis has developed results capable of predicting a feasible evolution of the studied process, it is possible to reverse the analysis problem. Thus, determining the input parameters, knowing the result of a finite element analysis, becomes an inverse problem, and is called the identification of parameters. This type of problem occurs in the study of the behavior of the material, so the problem is one of modeling and then a determination by numerical calculations, in most cases, of the material parameters that correspond to the final result already determined by solving the direct problem, Liu, W., Yang, Y. Y. \& Z. W. Xing, [3]. Another type of the problem is that of the initial geometry of the specimens or tool shape design so that the desired shape of the finished piece is finally obtained, Kleinermann, J.-P., Ponthot, J.-P., [4] 
1.1 Introduction to Optimization Study using Ansys®- DesignXplorer (Design Exploration module)

Design Exploration is a particularly proficient approach used by the DesignXplorer feature of Ansys ${ }^{\circledR}$ for exploring, understanding and optimization of technological processes and manufacturing technologies, namely in their design.

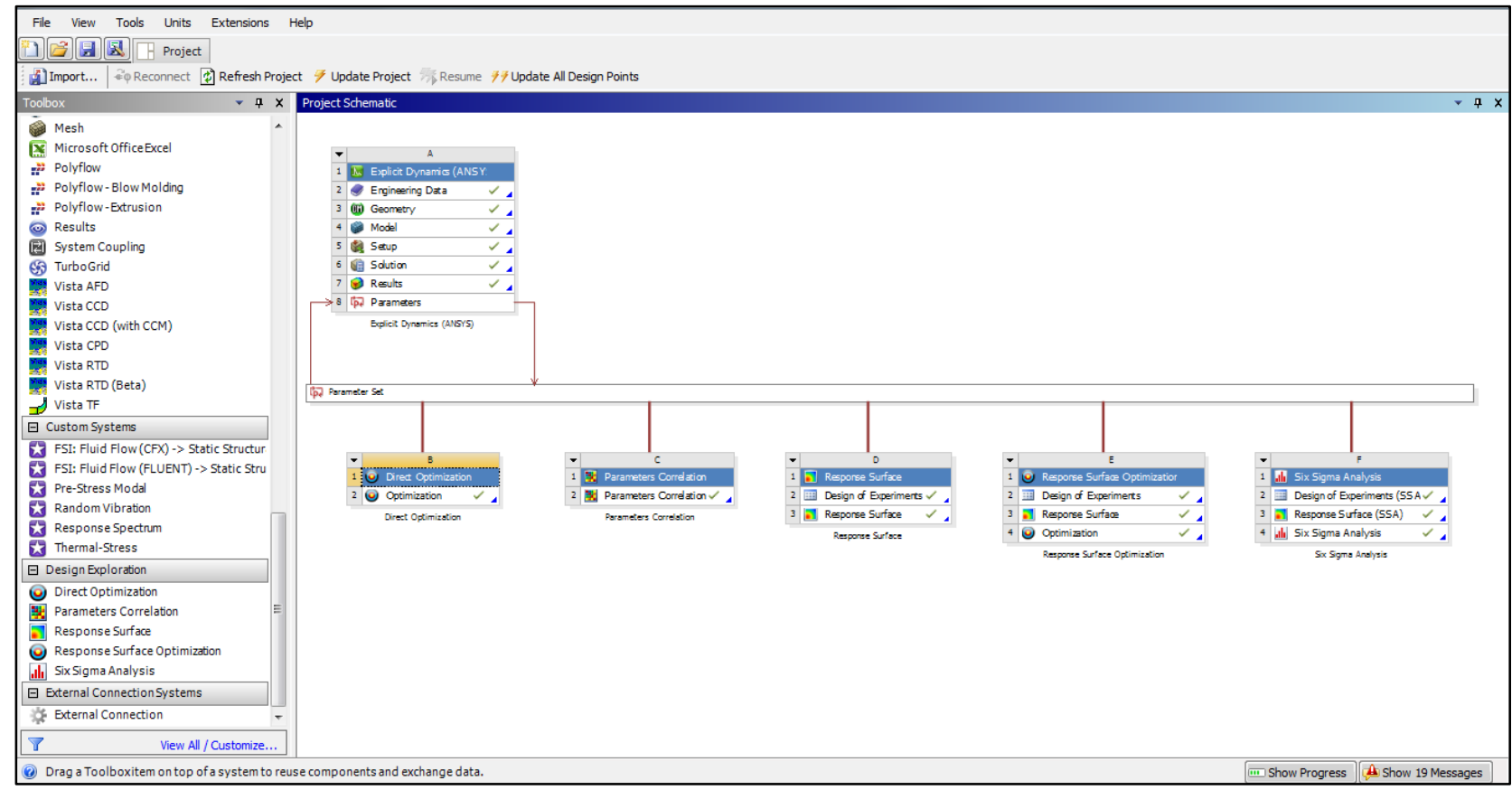

Fig. 1. The Project of Optimization General Scheme

The purpose of the Design Of Experiments (DOE) procedure is to collect a representative set of data relating to a process, technology, or an engineering project, adequate, data to calculate a response surface, and then executing an optimization (for optimization of a Response Surface too). A set of Design Points (DP) will be calculated. The Response Surface accuracy will depend plenteously on the DOE scheme adopted, and in particular, the number of Design Points have been computed.

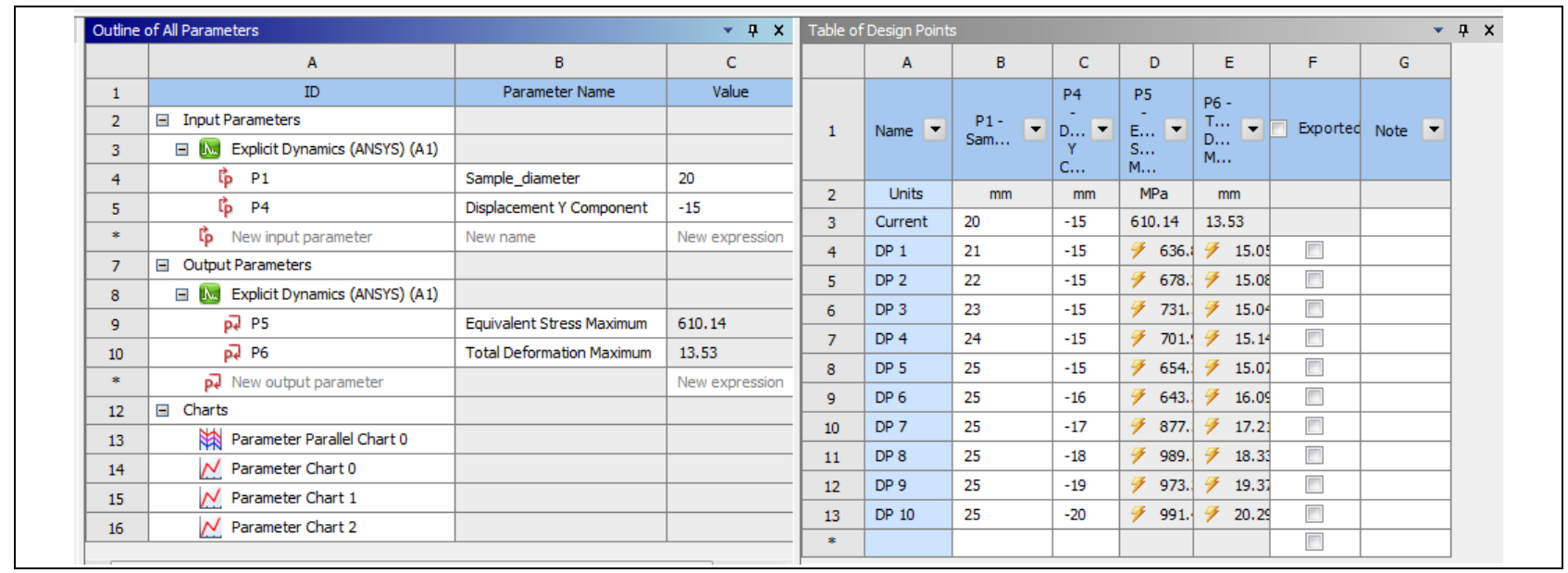

Fig. 2. Outline of All Parameters(left); Table of Design Points (right) 
Salem, N.: Parameterized Finite Element Analysis With Optimization of a Superpla...

DesignXplorer of Ansys ${ }^{\circledR}$ DOE proposes several schemes. Design points are selected automatically, default user space parameters for a specific project, so as to produce an efficient exploration of the area of interest:

- Central Composite Design (CCD) [default]

- Box-Behnken Design

- Optimal Space Filling Design

- Custom + Sampling

- Sparse Grid Initialization

- Latin Hypercube Sampling Design

\subsection{Direct Optimization}

Ansys ${ }^{\circledR}$ provides a constrained technique called Goal Driven Optimization (GDO), multi-objective optimization, where the best design is obtained from sets of given variants or objectives set and parameters constraints. Each type of GDO (Response Surface Optimization and Direct Optimization) system contains a component called Optimization, ANSYS Help Viewer [5]. The available optimization methods are(Fig. 4):

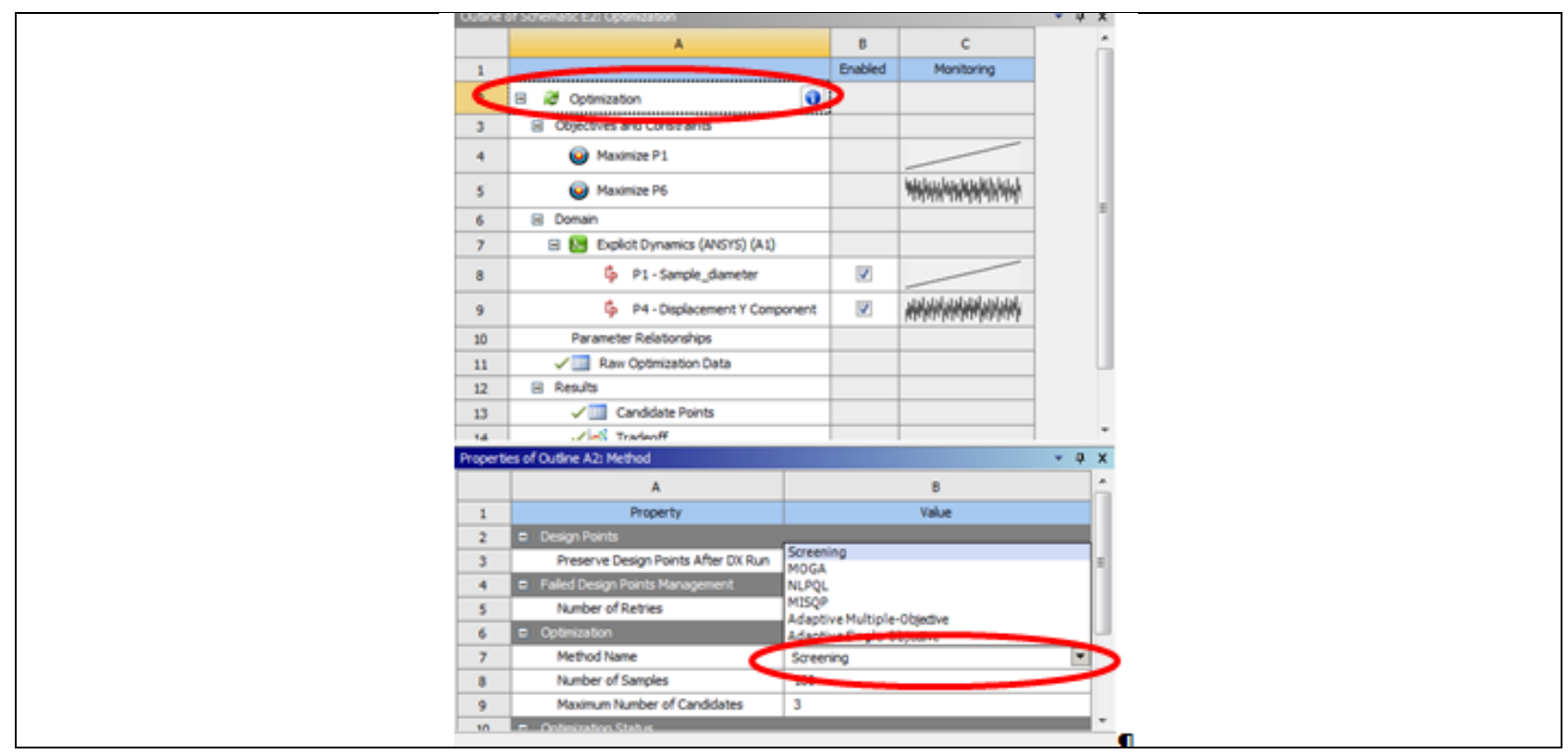

Fig. 3. The optimization methods available in Ansys ${ }^{\circledR}$

- Screening

- MOGA

- NLPQL

- MISQP

- Adaptive Single-Objective

- Adaptive Multiple-Objective

Screening, MOGA, and MISQP optimization methods can be used with discrete values. Screening, MISQP, MOGA, Adaptive Multiple Objective, and Adaptive Single-Objective optimization methods can be used for continuous values, also called Manufacturable Values. 
The Screening approach is a direct, non-iterative method to sampling by a quasirandom number generator based on the Hammersley algorithm. Note that Screening is used as a preliminary approach to sampling and evaluation. It should be considered as based on a random number generator; this consecutive method will use a new set of data for each approach.

\subsection{Optimization outline, (general theories; practice using Ansys)}

As optimization first step, DesignXplorer generates sampling:

$$
S(X, Y)
$$

subject to:

$$
\begin{aligned}
& g_{j}(X, Y) \leq 0 \quad j=1, \ldots, m \\
& X \in R^{N_{C}} \\
& Y \in R^{N_{i}} \\
& X_{L} \leq X \leq X_{U} \\
& Y_{L} \leq Y \leq Y_{U}
\end{aligned}
$$

The symbols $\mathrm{X}$ and $\mathrm{Y}$ represent vectors with the integer values of the continuous parameters, respectively. DesignXplorer allows the user to define a constrained design space with linear or non-linear constraints. The constraint sampling is a heuristic method based on Shifted-Hammersley (Screening) and Mixed-Integer Sequential Quadratic Programming (MISQP) sampling methods. MixedInteger Sequential Quadratic Programming (MISQP) is a mathematical optimization algorithm which solves Mixed-Integer Non-Linear Programming (MINLP) of the form, Exler, O. \& Schittkowski, K., [6]:

Minimize:

$f(x, y)$

subject to:

$g_{j}(x, y) \leq 0 \quad j=1, \ldots, m_{e}$

$g_{j}(x, y) \geq 0, j=m_{e}+1 \ldots m$

$x_{l} \leq x \leq x_{u}$

Where

$$
\begin{aligned}
& x \in \mathbb{R}^{n_{c}}, \quad y \in R^{n_{i}} \\
& x_{l} \leq x \leq x_{u} \\
& y_{l} \leq y \leq y_{u}
\end{aligned}
$$


The Multi-Objective Genetic Algorithm (MOGA) is a hybrid variant of the popular Non-dominated Sorted Genetic Algorithm-II (NSGA-II) based on controlled elitism concepts. It supports all types of input parameters, Wei, L. \& Yuying, Y., [7].

The Nonlinear Programming by Quadratic Lagrangian (NLPQL), is a mathematical optimization algorithm as developed by Schittkowski, K. [8]. This method is dedicated to solves constrained nonlinear programming problems of the form:

$$
\begin{aligned}
& \min f(x) \\
& g_{j}(x)=0, j=1 \ldots m_{e} \\
& x \in R^{n}: \\
& g_{j}(x) \geq 0, j=m_{e}+1 \ldots m \\
& x_{l} \leq x \leq x_{u}
\end{aligned}
$$

This computational procedure consists in an optimization scheme which generates a set of quadratic programming subproblems which are solved successively. Initially, this was a FORTRAN subroutine code and was included by Ansys ${ }^{\circledR}$, as optimization method option.

The Adaptive Single-Objective Optimization (ASO) is a mathematical optimization method that combines an Optimal Space-Filling (OSF) from Design of Experiments, a Kriging algorithm, Jakumeit; J., Herdy; M. \& Nitsche, M., [9], and the MISQP optimization algorithm. It is a gradient algorithm based on a response surface which provides a refined, global, optimized result.

Adaptive Multiple-Objective is a mathematical optimization method that combines the Kriging response surface and the MOGA optimization algorithm. This allows the user to generate a new set of data, or to use an old one, but with a more pronounced dispersion finishing. Except where necessary, this method does not evaluate all design points. In general, this method is a similar approach as MOGA, but a using a Kriging response surface, part of the population is "simulated" by Kriging and Kriging predictor error reduces the number of search line in finding the first Pareto front solutions. Adaptive Multiple-Objective optimization supports multiple objectives, multiple constraints, and is limited to continuous parameters and continuous parameters with Manufacturable Values.

In an engineering design process, it is very important to know what input parameters, and how many of these, are factors that influence in some way output parameters, and then decide which input parameters should be considered, Naceur, H., Guo, Y.Q. \& Batoz, J.-L., [10]. This is a lengthy process before you can produce a decision. Experiments designed to help revolutionize this long process of continuous testing and search engine design errors, and transforming it from a costly process into a statistical, efficient and powerful computational algorithm or method.

A very simple experiment is the Screening design. In this design, it is proposed a permutation of the lower limit and the upper limit (i.e., two levels) of each input parameter (factor), to study its effect on the output parameter that is of interest. It is obvious that this design, even if it is very simple and popular, at the same time, among 
industrial experiments, it only allows a linear effect if it exists between input parameters and output parameters. Thus, unfortunately, the effect of interaction between two or more parameters and output parameters can not be highlighted by this design. To compensate for this inadequacy of Screening design, it can be improved by including a center point of each input parameter within the experiments. This center point of each input parameter allows for a quadratic, minimum or maximum effect within the explored space, identifiable among input parameters and output parameters if they exist. This improvement is known as response surface design and allows a quadratic response model of responses estimated by optimization calculations. The quadratic mathematical response model can be calibrated using a full factorial design (all possible combinations of input parameters at each level) at three or more levels.

Moreover, this full factorial design requires more calculations and estimates than would normally be needed to outline a more accurate model of the parameters. As a deficiency of this design, it is to be said that the statistical procedure applied here in this design, with the aim of it being considered efficient, applies a technique using three or five levels, but not all possible combinations, known in the literature as Fractional factorial designs. Among the most popular fractional factorial designs for optimization models in the response surface design category are Central Composite Designs (CCD) and Box-Behnken Designs (BBM).

\subsection{Central Composite Designs(CCD)}

This feature is one of most used from that which is available for optimization model in the response surface category of design. This is an implicitly pre-set option, Rahman, M.M., [11].

Details about superplasticity and superplastic forming of this practical experiments are given in the literature. To perform a superplastic forming controlled process, the sample plate is clamped between the two components of the forming die, Grebenisan G. \& Muresan S., [12], on its perimeter and the assembly is heated in a horizontal oven, to high forming temperature (about half the melting temperature of the material, i.e. approximately $460\left[{ }^{\circ} \mathrm{C}\right]$, for Supral 100 , commercial named Aluminium alloy. After the temperature regime is reached a gas pressure will deform the specimen with a very low strain rate $\varepsilon^{\circ}=2.5 \times 10^{-3}\left[s^{-1}\right]$. The pressure inside to the die is automatically updated so as to keep a maximum value for the equivalent viscoplastic strain rate above nominated. The material is modeled with an Anand

Viscoplasticity law as described in Rahman, M.M., [11]. The strain distribution on the workpiece section and resultant thickness of the specimen it's possible to be very variable from one area to another, leading to a nonhomogeneous thickness distribution in the final component. This will also induce higher stress zones. The authors Ponthot, J.-P., Kleinermann, J.-P., [13] proposed an approach to avoid these problems, and they proposed to use the following procedure: to determine the initial piece geometry that would lead, at the end of forming process to the prescribed uniform distribution of the thickness in the final workpiece transversal section. Furthermore, we chosen as input parameters the sample diameter and displacement of the sample inside the die, during the forming process, respectively, Kim, NamHo., Choi, K. K. \& Chen, J. S., [14]. 
Salem, N.: Parameterized Finite Element Analysis With Optimization of a Superpla...

\subsection{Design of Experiments(DOE)}

A DOE study involves a sampling point not always conducive to lowering the time needed for the analysis. For example, applying a CCD in a fractional factorial scheme, a dozen times more sampling points are required for a number of input parameters, that is, as many evaluations as Finite Element Analysis, which means a very large computational time is consuming, Gantar, G., Pepelnjak, T. \& Kuzman, K [15].

Therefore, it is necessary to perform a non-important input parameter removal operation when possible. This removal can be accomplished by a tool called correlation matrix. The correlation matrix helps users identify input parameters considered as nonimportant and treated as deterministic parameters in the SSA study, fig. 5:

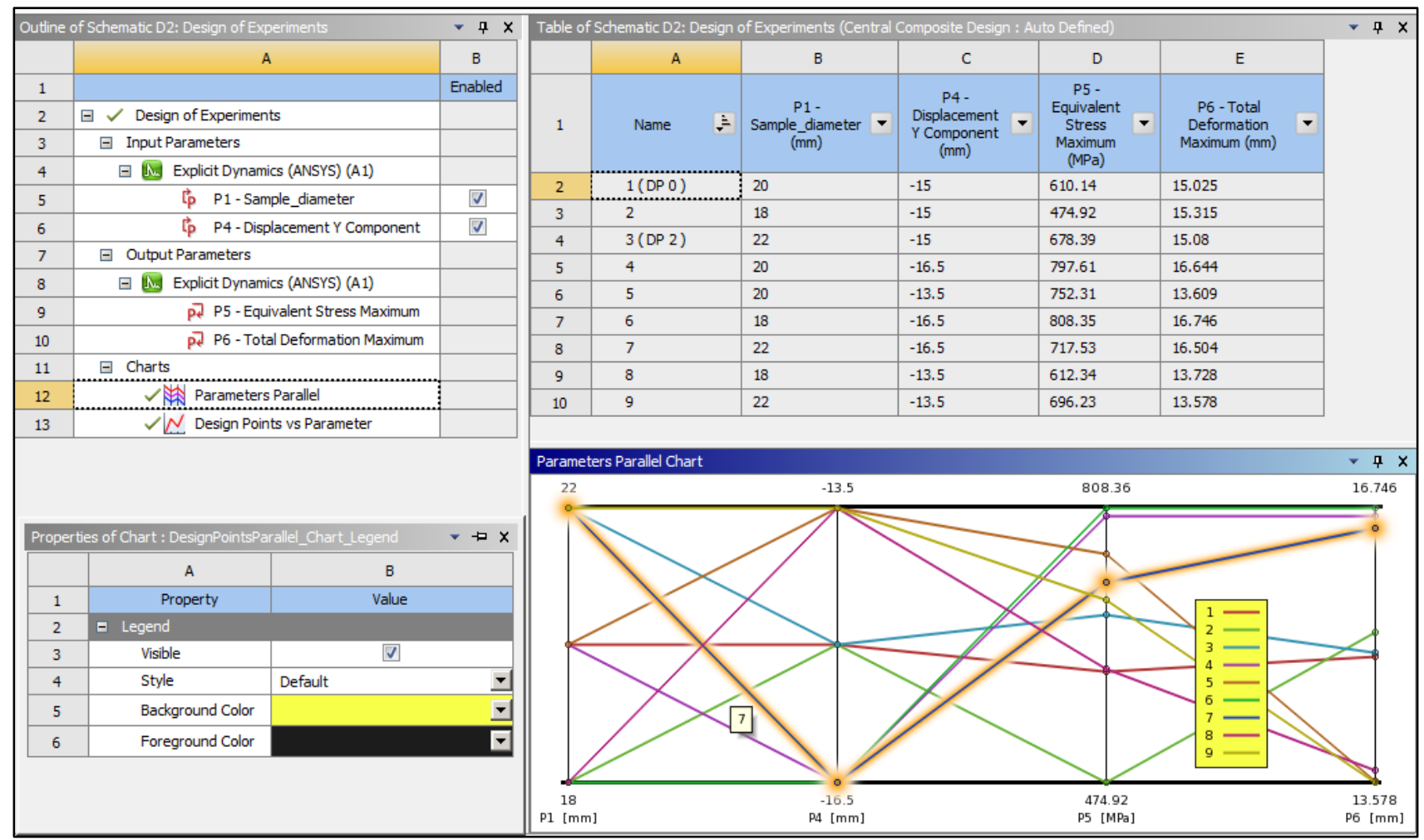

Fig. 4. Design of Experiments features and results

\subsection{Parameters Correlation Matrix}

A Parameters Correlation study allows two very important things:

- which input parameters have the greatest impact on design;

- identifies how the input-output relationship becomes linear or quadratic.

These responses can be easily obtained as the study offers an excellent graphical approach through the Parameters Correlation Charts.

The finite element analysis has performed, and the influence and impact of the input parameters to the output parameters is described in the following charts, fig. 6 : 


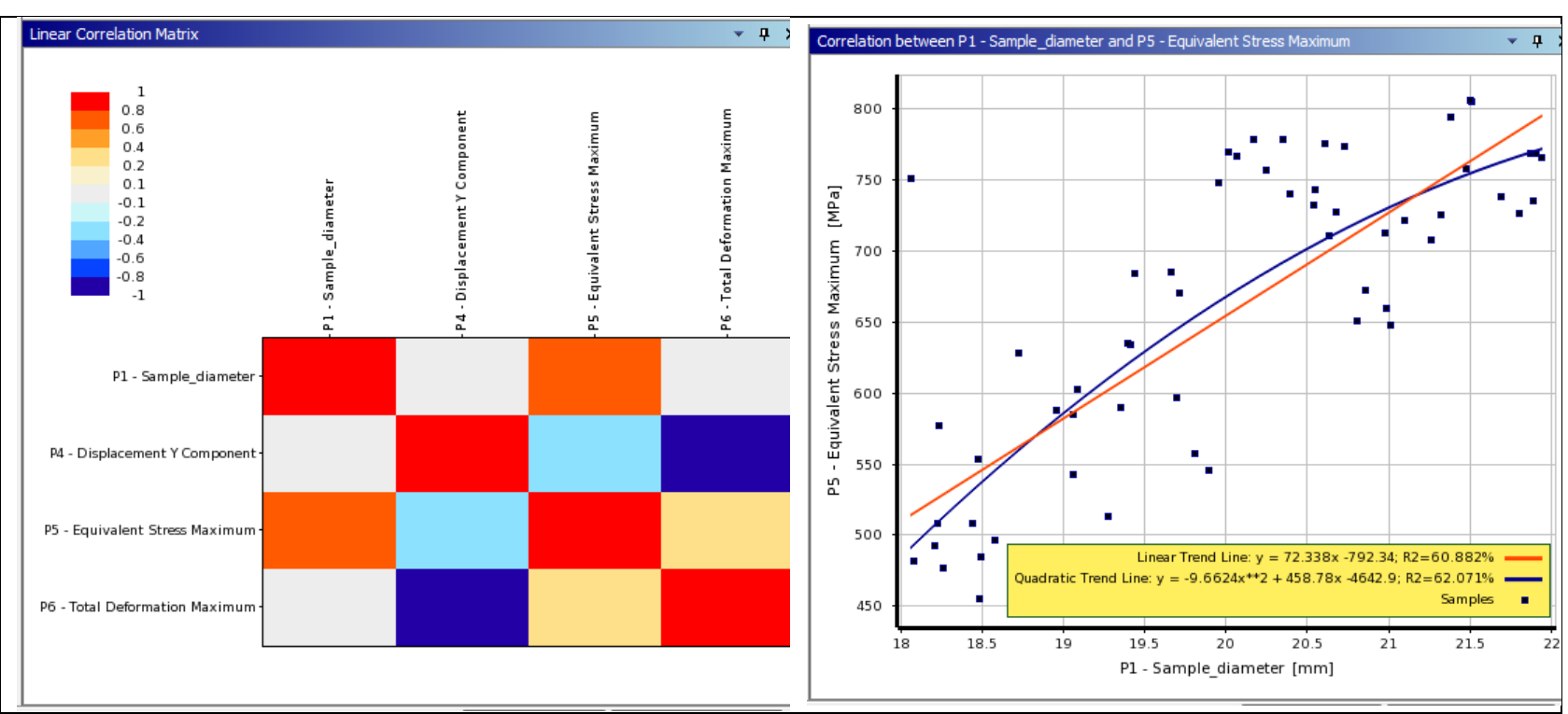

Fig. 5. Correlation Matrix and Correlation Scatter

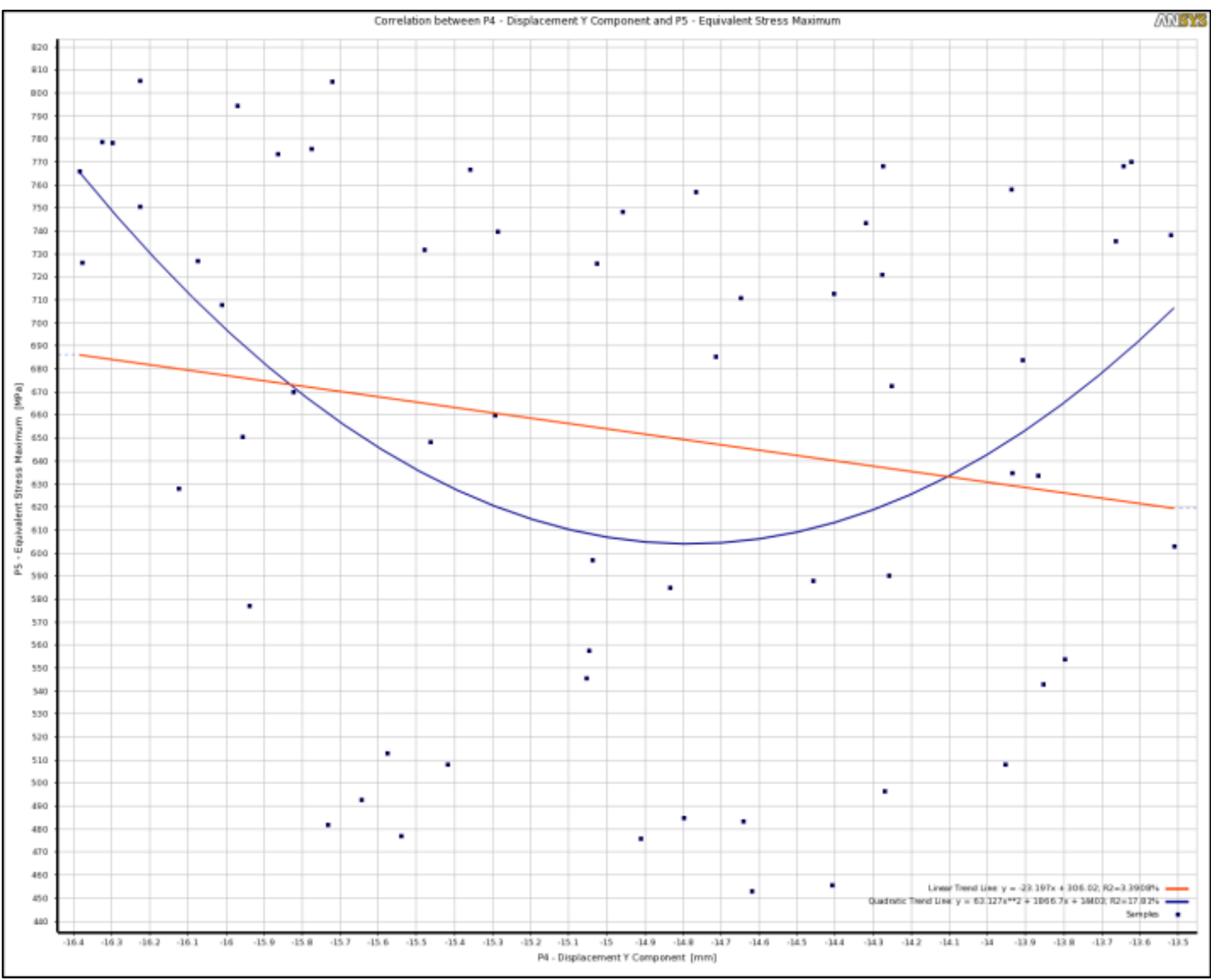

Fig. 6. Correlation (Scatter) between Displacement and Equivalent Stress Maximum(von Mises) 
Salem, N.: Parameterized Finite Element Analysis With Optimization of a Superpla...

The fig. 7 shows a distinguished influence of Sample_Diameter input parameter on Equivalent Stress Maximum, which gives us the measure of involving of sample diameter on the shape of the finite workpiece, and the chance of crack appearance, the local necking evolution.

Whereas we expected that the influence of displacement magnitude changing would influence the thickness variations and the uniform distribution, nevertheless, this input parameter, i.e.

Displacement Y Component, don't have an outstanding implication on process output parameter, as Equivalent Stress $\quad$ Maximum, fig. 7. Figure 7 shows a wide spread of value founded and computed at each evaluation, i.e. at each Finite Element Analysis.

\section{Response Surface Optimization}

By performing GDO or Six Sigma Analysis(SSA), the optimization approach, after a Finite Element Analysis has been performed, is recommended after a Design of Experiments (DOE) study has been performed.

In this way, Ansys ${ }^{\circledR}$ provides the operator with a response surface, fig. $\left.8, a, b\right)$, and the response surface optimization samples, fig. 9 (image shows the Sample 6144 from 10000 evaluations), built into the area of interest for the project, based on the design points chosen, Donglai, W., Zhenshan, C. \& Jun, Ch., [16], Raz, K.; Cechura, M. \& Chval, Z. [17].

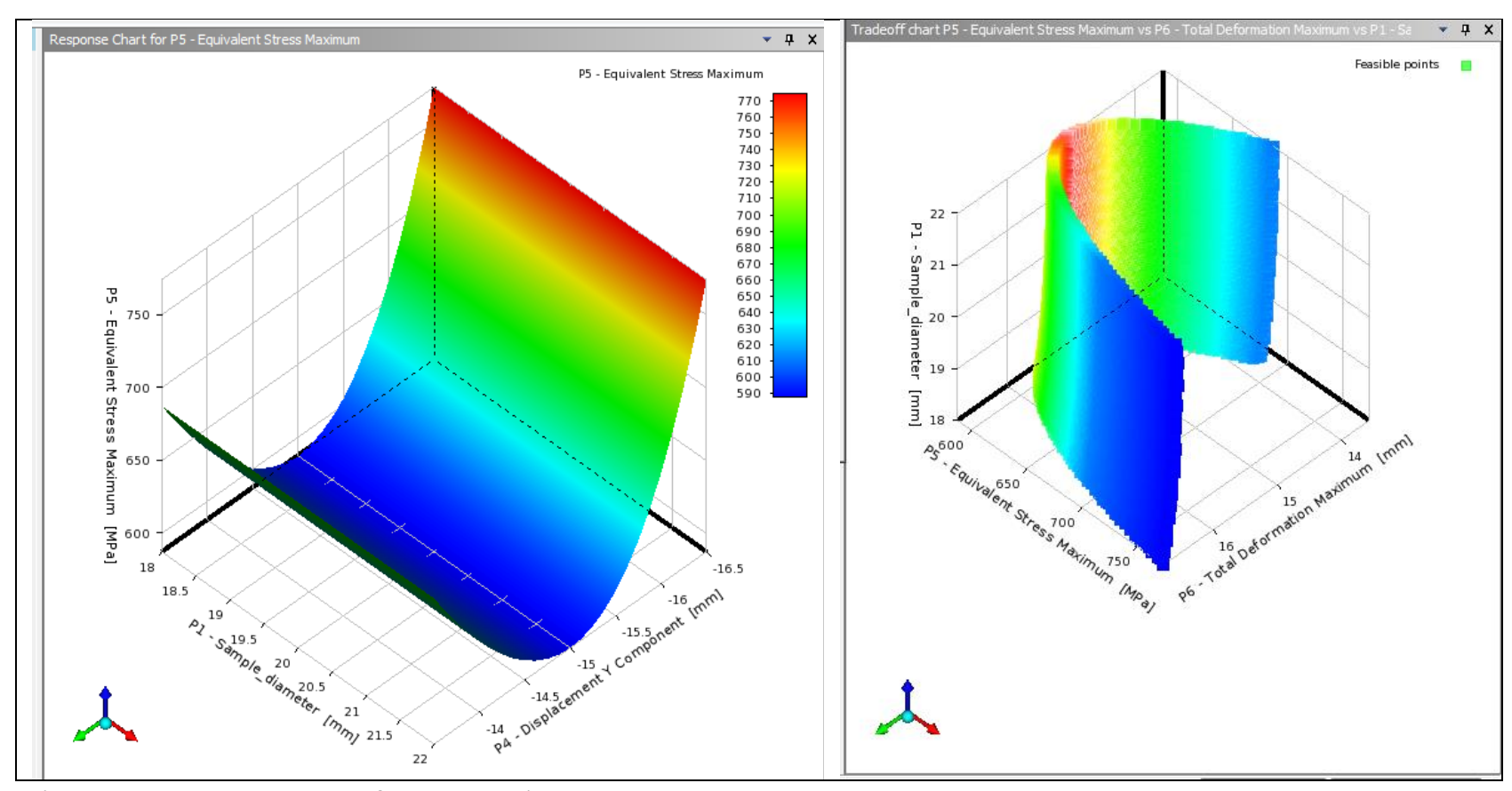

Fig. 7. Response surface results 


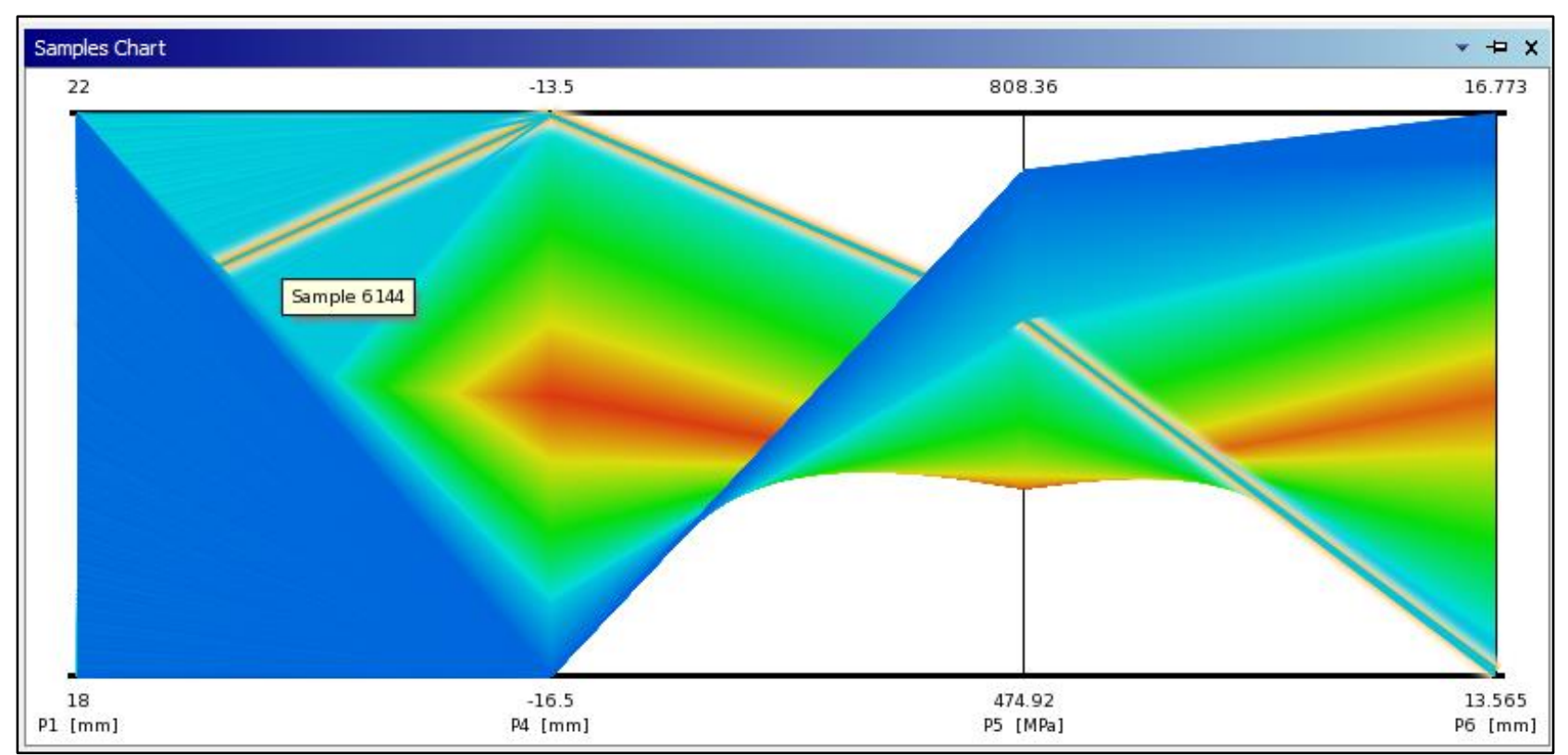

Fig. 8. Response Surface Optimization-Samples

\section{Six Sigma Analysis}

Six Sigma Analysis allows the user to determine, based on Gaussian dispersion, uncertainties in a pattern. An uncertainty can be a parameter or the value of this parameter at a given time. This type of analysis allows based on the Six Sigma Analysis quality criterion to determine with some precision if a design has a certain parameter within the quality limits of the normal Gaussian distribution. Generally, a parameter for controlling the model's quality specification is proposed as an output parameter, the values of which must fall within two limits: the lower limit or the upper limit of the quality criterion

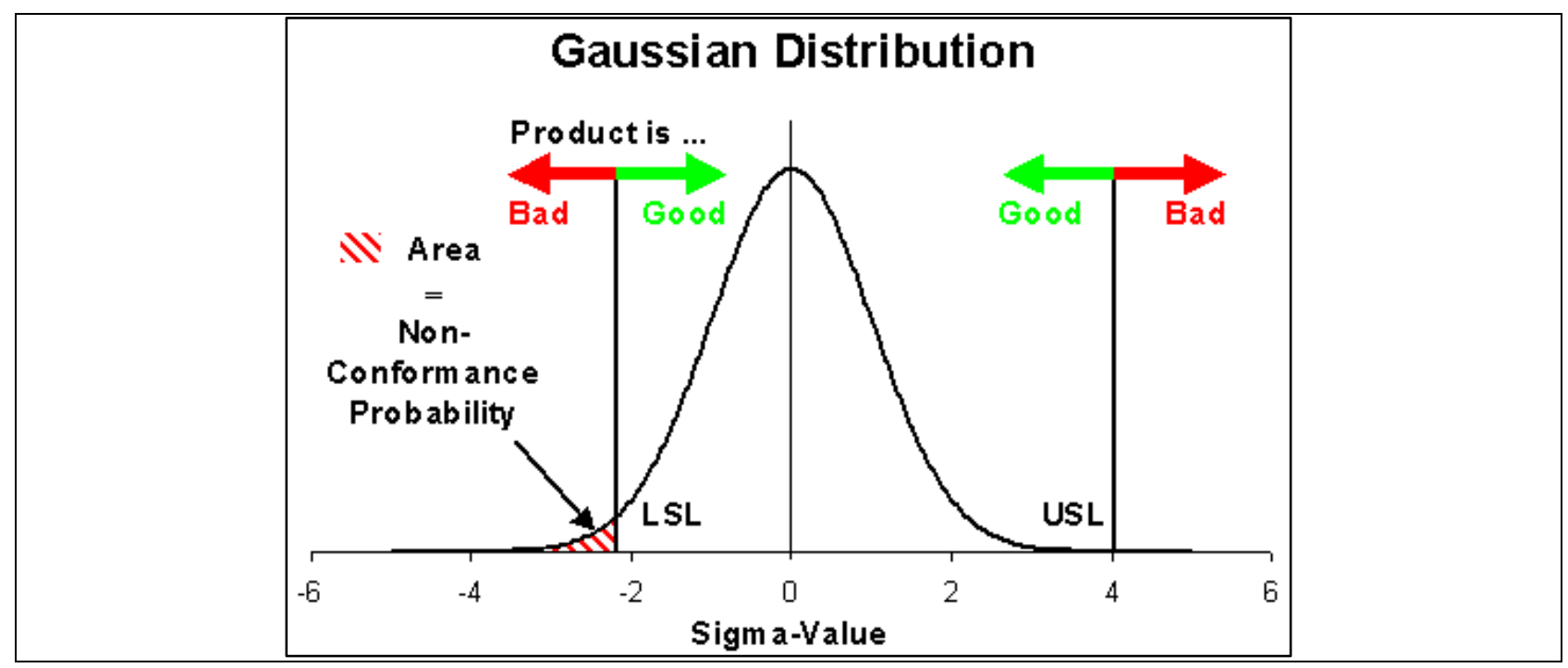

Fig. 9- The Gauss distribution, Ansys ${ }^{\circledR}$ Help Viewer [5]

-LSL $=$ lower specification limit $-\mathrm{USL}=$ upper specification limit 
In industrial practice, it rarely happens that an output parameter follows a normal Gaussian distribution. By definition, Six Sigma Analysis represents the normal, symmetric Gauss distribution for which the distribution width is of an absolute value of 6 sigma $(\underline{ \pm \sigma})$, that is, the deviation from the mean value is $\underline{3 \sigma}$.

For distributions other than Gauss, it is considered acceptable for those distributions for which the definition is valid: 3.4 units per million are out of the standard distribution. The SSA results are based on computing nine design points values for each input and output parameters, respectively, fig. 11, a, b), also the surface response built for input and output parameters, fig. 12:

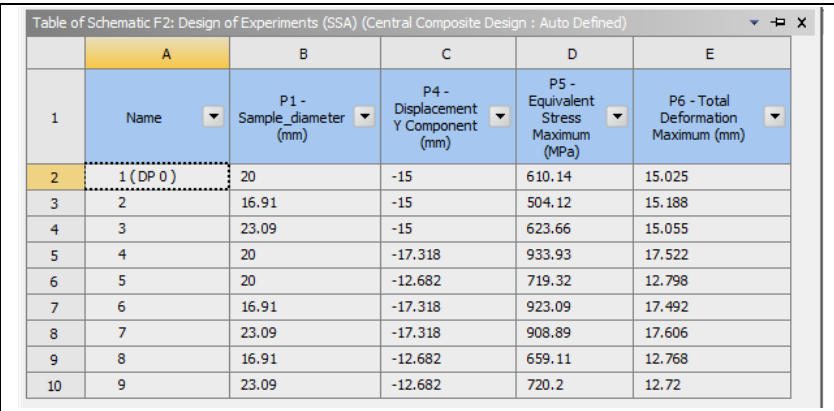

a)

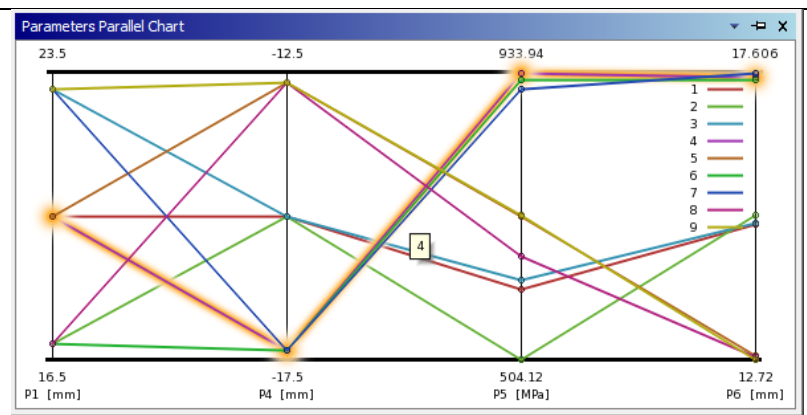

b)

Fig. 10. Design of Experiments computed for Six Sigma Analysis

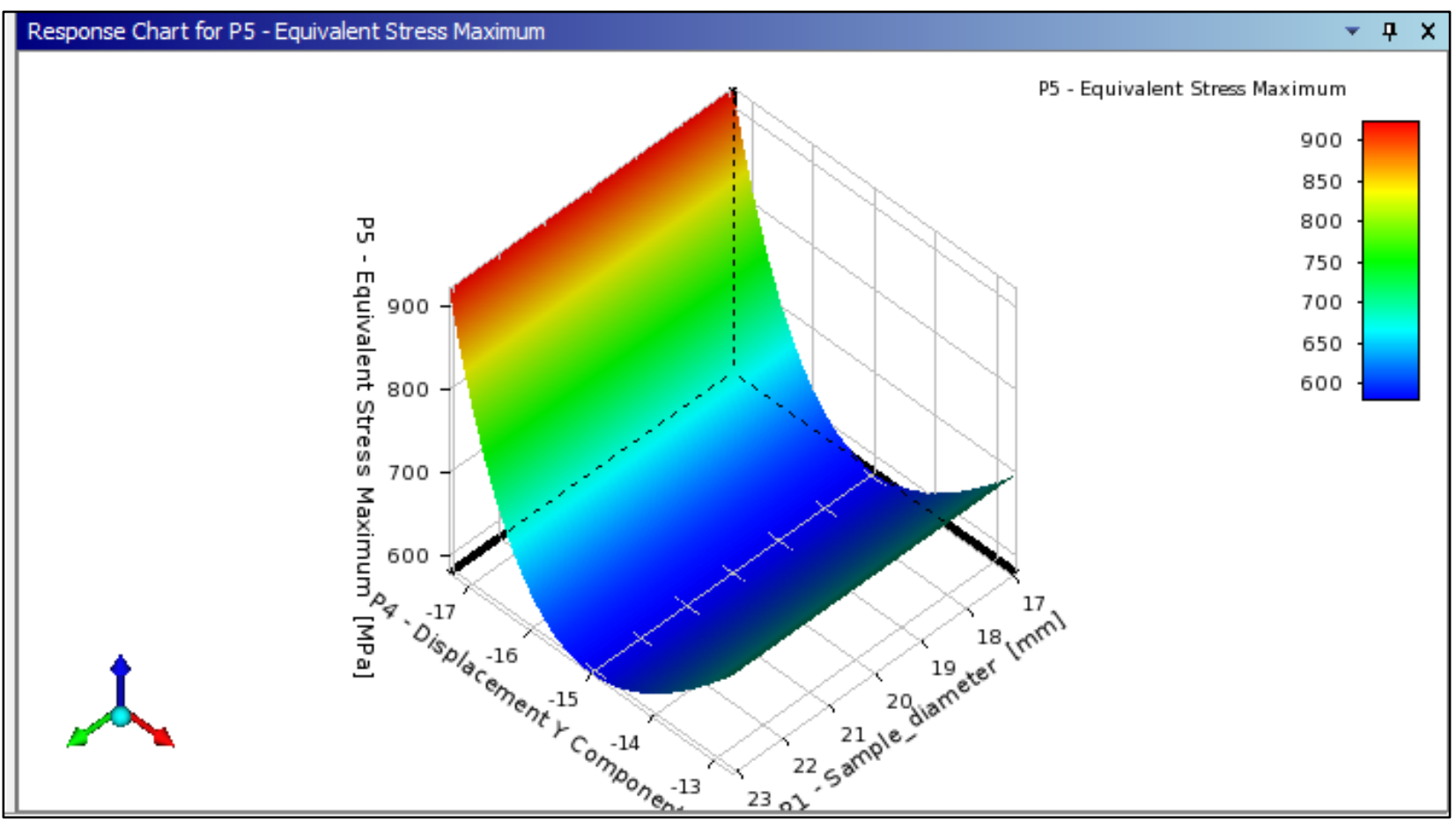

Fig. 11. Response Surface built for Six Sigma Analysis

\section{Conclusion}

The engineering system design selects one or more variables that meet a set of objectives. A better design can be obtained if, using certain algorithms, a certain cost function can be reduced. The design is optimal when the lowest cost of all feasible models can be obtained for a particular model. Almost always, design choices are 
limited due to resource constraints, such as material and work constraints, as well as physical and other restrictions. A feasible area within a design space is circumscribed by boundary boundaries. More importantly, both cost function and constraints can be expressed as mathematical functions involving design variables. The resulting mathematical optimization problem can then be resolved using different optimization methods.

If we are going to introduce a typical optimization problem in engineering design, it should include the following steps: setting the problem and describing it; a preliminary data collection investigation as a pre-wording; identifying design variables; design criteria and constraints; the mathematical formulation of the optimization problem; finding the solution to the proposed problem.

Engineering design optimization is an open-ended problem. The most important step in the optimization problem is the correct mathematical formulation of the problem, i.e., the mathematical optimization model. Once the problem has been formulated, a wide variety of analytical and computational methods are available to find a solution. Applying Goal Driven Optimization and Six Sigma Analysis to analysis, or design optimization study, is always a challenge in terms of saving time, especially where design complexity is high. This obviously implies a very laborious analysis by finite element method. The same can be said about a project in which the technological parameters are non-linear, or the material has a non-linear behavior.

\section{References}

Diehl, M., Glineur, F., Jarlebring, E. \& W. Michiels, (2010). Recent Advances in Optimization and itsApplications in Engineering, Springer,

Belegundu, A. D. \& Chandrupatla, T. R. (1999). Optimization Concepts and Applications in Engineering, Prentice Hall

Liu, W., Yang, Y. Y. \& Z. W. Xing, (2005). Automatic Process Optimization Of Sheet Metal Forming With Multi-objective, Volume A, Numisheet 2005, edited by L. M. Smith, F. Pourboghrat, J. -W. Yoon, \& T. B. Stoughton, American Institute of Physics 0-7354- 0265-5/05,

Kleinermann, J.-P., Ponthot, J.-P., (2003). Parameter identification and shape/process optimization in metal forming simulation, Elsevier Science B.V., Journal of Materials Processing Technology 139 521-526, 2003.

Ansys ${ }^{\circledR}$ manual, (ANSYS $₫$ Help Viewer)

Exler, O. \& Schittkowski, K., (2007). A trust region SQP algorithm for mixed-integer nonlinear programming, Optimization Letters, Vol. 1, p.269-280, 2007.

Wei, L. \& Yuying, Y., (2008). Multi-objective optimization of sheet metal forming process using the Pareto-based genetic algorithm, Elsevier Science B.V., Journal Of Materials Processing Technology 208, 499-506.

Schittkowski, K., (1985). NLPQL: A FORTRAN subroutine solving constrained nonlinear programming problems, Annals of Operations Research 5(1985/6)485 500. Jakumeit; J., Herdy; M. \& Nitsche, M., (2005). Parameter optimization of the sheet metal forming process using an iterative parallel Kriging algorithm, Struct Multidisc Optim 29: 498. doi:10.1007/s00158-004-0455-3. 
Naceur, H., Guo, Y.Q. \& Batoz, J.-L., (2004). Blank optimization in sheet metal forming using an evolutionary algorithm, Elsevier Science B.V., Journal of Materials Processing Technology 151 183-191.

Rahman, M.M., (2011). Optimization of Process Parameters on Ti-6Al-4V Using Central Composite Design Method, Advanced Materials Research, Vols. 189-193, pp. 1393-1400.

Grebenisan G. \& Mureşan, S., (2007). Numerical Study Of Volumic Forming On Superplastic Behaviour, Annals of The University of Oradea. Fascicle of Management and Technological Engineering, Volume XVI (VI), ISSN 1583-0691, DOI: 10.15660/AUOFMTE.2007.714.

Ponthot, J.-P., Kleinermann, J.-P., (2006). A cascade optimization methodology for automatic parameter identification and shape/process optimization in metal forming simulation, Elsevier Science B.V., Comput. Methods Appl. Mech. Engrg. 195, 54725508.

Kim, NamHo., Choi, K. K. \& Chen, J. S., (2001). Die shape design optimization of sheet metal stamping process using meshfree method, Int. J. Numer. Meth. Engng; 51:1385-1405 (DOI: 10.1002/nme.181).

Gantar, G., Pepelnjak, T. \& Kuzman, K (2002). Optimization of sheet metal forming processes by the use of numerical simulations, Elsevier Science B.V., Journal of Materials Processing Technology 130-131, 54-59.

Donglai, W., Zhenshan, C. \& Jun, Ch., (2008). Optimization and tolerance prediction of sheet metal forming process using response surface model, Elsevier Science B.V., Computational Materials Science 42, 228-233.

Raz, K.; Cechura, M. \& Chval, Z. (2016). Upper Crossbeam of Free Forging Press Optimization in Order to Improve Mechanical Properties and Durability, Proceedings of the 26th DAAAM International Symposium, pp.0524-0529, B. Katalinic (Ed.), Published by DAAAM International, ISBN 978- 3-902734-07-5, ISSN 1726-9679, Vienna, Austria DOI: 10.2507/26th.daaam.proceedings.071. 